\title{
GaAs(110) surface electronic structure by metastable deexcitation spectroscopy
}

\author{
L. Pasquali* and S. Nannarone \\ Istituto Nazionale di Fisica della Materia, unità di Modena \\ and Department of Physics, University of Modena, via Campi 213/a, 41100 Modena, Italy \\ M. Canepa and L. Mattera \\ Istituto Nazionale di Fisica della Materia, unità di Genova \\ and Department of Physics, University of Genova, via Dodecanneso 33, 16146 Genova, Italy
}

(Received 17 July 1995)

\begin{abstract}
Metastable deexcitation spectroscopy was applied to study the surface valence electronic structure of clean cleaved $\mathrm{GaAs}(110)$. Metastable deexcitation spectroscopy was flanked by angle-resolved photoemission. An effective surface density of states was derived from the experimental spectrum through deconvolution. Two groups of states were observed in the 0-4 and 5-8 eV range of binding energy, respectively. These features were ascribed to emission from surface states. A plane-by-plane tight-binding density-of-states calculation was performed. More quantitative insights were obtained by comparing experimental and theoretical results. The most prominent feature of the first group of states of deconvolution was assigned to surface state $A_{5}$. Contributions from states $A_{4}, A_{3}, A_{1}^{\prime}$, and $A_{2}^{\prime}$ were also observed. The doublet of the second group of features was identified with $C_{2}$ and $C_{1}$. Relative amplitudes of effective surface density of states were related to surface charge density.
\end{abstract}

\section{INTRODUCTION}

Determination of surface and interface electronic structure is one of the main issues in modern surface physics. Infrared optical spectroscopy, ${ }^{1}$ photoemission spectroscopy (UPS), ${ }^{2}$ electron energy loss spectroscopy, ${ }^{3}$ and inverse photoemission spectroscopy, ${ }^{4}$ have been widely used over a period of about twenty-five years to obtain energy, wave vector dispersion, and the density of full and empty surface states of clean surfaces and interfaces.

Besides this, another crucial property of surface and interface orbitals is charge density, knowledge of which is basic to the understanding of surface geometry, surface reactivity, and surface and interface bonding. However, in spite of this important role, theoretical calculations are to date the main and indeed almost the only source of information on surface charge density (SCD). From an experimental point of view, orbital resolved information on SCD can be obtained only by means of a few techniques. Extremely interesting results have been obtained with scanning tunneling microscopy, which probes the surface charge with limitation to conducting materials. In semiconductors, its use has been generally restricted to orbitals occurring at energies close to a band edge. The $(e, 2 e)$ coincidence spectroscopy gives the Fourier transform of the orbitals. The technique is well established for gases, while its application to surfaces is still at a pioneering stage. ${ }^{5}$

As far as valence surface density of states (SDOS) is concerned, to date the main body of information has been supplied by photoemission, where the contributions from the first surface atomic plane are in competition with deeper excited planes contained in the escape depth.
Among the spectroscopies, where the excitation is confined to the surface plane, positron-annihilation-induced Auger-electron spectroscopy has shown interesting results with high surface specificity. ${ }^{6}$

Besides this, following the pioneering work carried out by Hagstrum and co-workers, other spectroscopies based on beams of ionized or excited neutral noble atoms as probes, such as ion neutralization spectroscopy (INS) Refs. 7 and 8 or metastable deexcitation spectroscopy (MDS) ${ }^{9}$ have proven to be extremely sensitive to the outermost features of surface electronic structure. Although the two techniques are very similar from the point of view of physics and nature of information, MDS has prevailed over INS over the years, due to the fact that it does not involve the experimental difficulties connected with low energy ion beam focusing, it is completely undestructive, and it is believed to suffer less from broadening effects. ${ }^{10}$

In spite of its being extremely surface sensitive and nondestructive, only a small amount of research on semiconductor surfaces has been based on MDS. An INS study of the $\operatorname{GaAs}(110)$ surface was published by Pretzer and Hagstrum in $1966,{ }^{11}$ but information on surface electronic structure was not extracted therefrom. MDS spectra of $\operatorname{Si}(111) 7 \times 7$ and $\operatorname{Si}(100) 2 \times 1$ were presented by Masuda et al. ${ }^{12}$ without attempting an in-depth analysis in terms of SDOS.

Presumably, one of the main reasons for this lack of exploitation of MDS on clean semiconductor surfaces can be ascribed to the nature of the deexcitation mechanism on this kind of surface. In fact, because of the value of the semiconductor electron affinity, the most probable deexcitation process is a resonant ionization (RI) followed by an Auger neutralization ( $\mathrm{AN}),{ }^{7}$ as schematically shown in Fig. 1 (RI+AN). This deexcitation mechanism leads to an energy distribution of emitted electrons, which can 


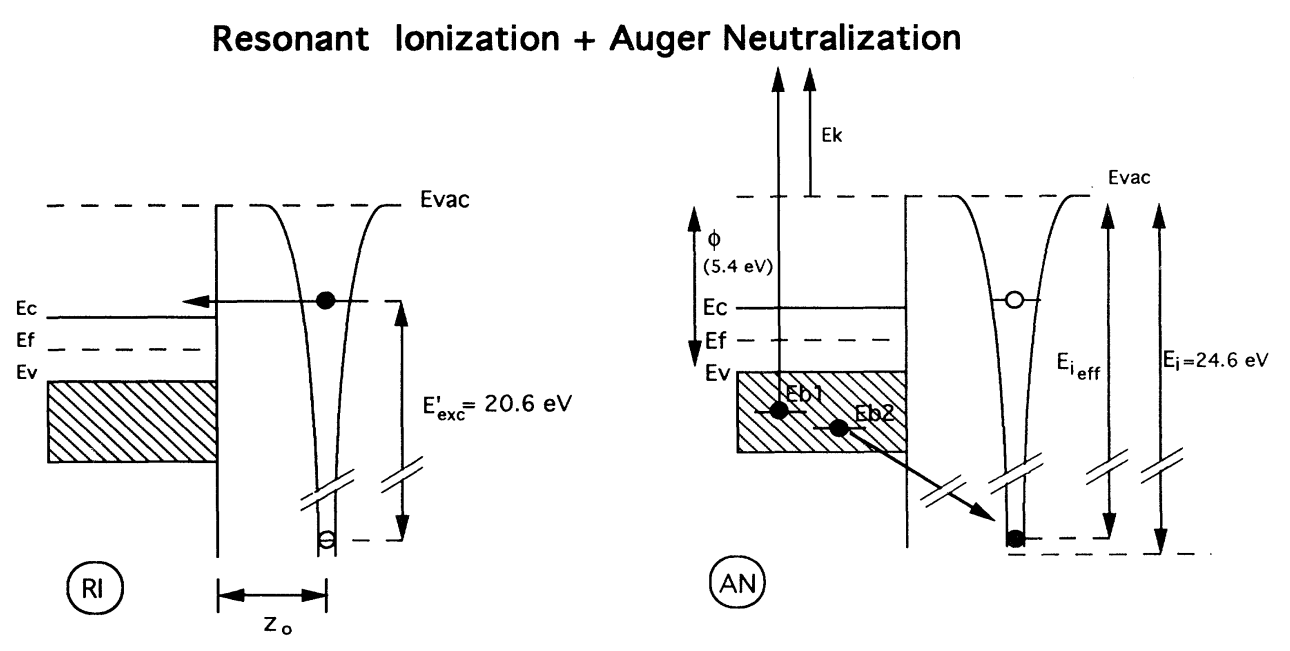

FIG. 1. Electron-energy diagram for incident metastable He atoms undergoing a RI+AN deexcitation in front of a semiconductor surface. The resonant ionization of the metastable atom (on the left) takes place when the excited occupied state of the atom is degenerate in energy with empty states of the surface available for tunneling and at the distance $z_{0}$, at which the wave functions begin to overlap significantly. The Auger neutralization process (on the right) involves two surface valence band electrons and the $1 s$ hole of the incoming atom. The emitted electrons reflect, in first approximation, the self-convolution of the SDOS projected on the He atom.

be related to the self-convolution of the SDOS weighted by the AN matrix element, whose value depends on the features of the SCD. Consequently, SDOS is obtained from the experimental spectrum through a mathematical deconvolution. ${ }^{13}$

In the present paper, MDS is applied, in a systematic and extensive way, to the study of a prototype semiconductor surface [clean $\mathrm{GaAs}(110)$ ], in order to achieve an insight into surface electronic valence band structure (SDOS and SCD). The present study is based on MDS, flanked by UPS, and on a comparison with theoretical calculations.

Present results bear importance in fundamental surface semiconductor physics and, at the same time, serve as a basis for any spectroscopical study of low dimensional systems based on III-V compounds, such as multilayer structures and multiple quantum wells, of modern device technology.

The paper is organized as follows. In Sec. II a brief account of the RI+AN deexcitation of a metastable atom $A^{*}$ in front of a semiconductor surface is given. The experimental technique is described in Sec. III, while data are presented and discussed in Sec. IV. Conclusions are drawn in Sec. V. In the Appendix, a brief description of the deconvolution procedure adopted is given.

\section{PHYSICS OF THE RI+AN PROCESS}

In this section, the physics of the $\mathrm{RI}+\mathrm{AN}$ deexcitation process in front of a semiconductor surface, developed in Refs. 7 and 8 , is briefly outlined in order to give the basic and essential elements for the analysis and for the understanding of the experimental results.
The process is described by the following reaction:

$$
A^{*}+S \longrightarrow A+S^{+}+e^{-},
$$

where $A^{*}$ and $A$ indicate the atom in the metastable and ground state, respectively, $S$ is the semiconductor surface, and $e^{-}$the emitted electron. The kinetic energy, $E_{k}$, of the emitted electron referred to the vacuum level is given by

$$
E_{k}=E_{i_{\text {eff }}}-\left[E_{b 2}+\left(\chi+E_{\mathrm{gs}}\right)\right]-\left[E_{b 1}+\left(\chi+E_{\mathrm{gs}}\right)\right],
$$

where $E_{b 1}$ and $E_{b 2}$ are the binding energies of the two semiconductor electrons involved, $\chi$ is the electron affinity, and $E_{\mathrm{gs}}$ the main energy gap at the surface. Finally, $E_{i_{\text {eff }}}$ is the effective ionization energy of the He $1 s$ atomic electron in front of the surface. Its value can be estimated by taking into account the fact that the interaction between the ion and the surface mainly occurs through the image charge potential. ${ }^{14}$ The calculation gives

$$
E_{i_{\mathrm{eff}}}=E_{i}-\frac{(\epsilon-1) 3.6}{(\epsilon+1) z_{0}}
$$

where $z_{0}$ is the most probable distance at which deexcitation occurs and $\epsilon$ the semiconductor dielectric constant, whose value for $\mathrm{GaAs}$ is $\epsilon_{\mathrm{GaAs}}=13.1 .^{15}$

The position of the features of the kinetic energy distribution, $N\left(E_{k}\right)$, of the emitted electrons, together with their intensities, can be related to the SDOS and SCD. In fact, $N\left(E_{k}\right)$, under a reasonable hypothesis (see Ref. 13 and references therein) has the same peaks of the selfconvolution, $P(E)$, of the SDOS weighted by the matrix element of the AN process, $W_{\mathrm{fi}} . \mathrm{W}_{\mathrm{fi}}$ is related to the extent of superposition between the $\mathrm{He} 1 s$ and the surface 
orbitals and thus it is related to the characteristics of the SCD.

Consequently $P(E)$ can be written as

$$
P(E) \propto \int\left\|W_{\mathrm{fi}}\right\|^{2} \rho_{z_{0}}(E-x) \rho_{z_{0}}(E+x) d x,
$$

where $\rho_{z_{0}}(\mathrm{E})$ is the SDOS at $z_{0}$ and $E$ varies through the valence band. $P(E)$ is related to $N\left(E_{k}\right)$ through the change of variable,

$$
E=\frac{E_{i_{\text {eff }}}\left(z_{0}\right)-2\left(\chi+E_{\mathrm{gs}}\right)-E_{k}}{2}
$$

In order to derive information on SDOS from the experimental results, two approaches are possible: direct and inverse. The direct approach consists of comparing the experimental spectrum with simulations based on properly calculated theoretical SDOS; though being the most promising, it was used only in a few cases (see, for example, Ref. 16). Instead, the inverse one consists of deriving the SDOS through a mathematical deconvolution of the experimental spectrum. This is the chosen approach for the present work.

\section{EXPERIMENT}

The experiment was run in the ultrahigh vacuum experimental system described in Ref. 17 at a base pressure of $\sim 5 \times 10^{-11}$ Torr. Clean surfaces were prepared by cleavage.

The metastable atom source used in this experiment is described in Ref. 18. Briefly, the supersonic beam is obtained by gas (helium) expansion from 7 atm to $10^{-3}$ Torr through an aperture of $20 \mu \mathrm{m}$ diameter. Collimation is achieved by a skimmer of $0.5 \mathrm{~mm}$ diameter and a 3 $\mathrm{mm}$ aperture $10 \mathrm{~cm}$ from the sample surface. Excitation is obtained by an electron gun coaxial with the beam operated at an energy of $400 \mathrm{eV} \cdot{ }^{19}$ In this way $90 \%$ of excited helium atoms are estimated to be in singlet state. Beam intensity is $\sim 10^{7}$ atoms per second at the sample surface.

The beam impinged at $45^{\circ}$ with respect to the sample normal. Electrons were detected and energy analyzed by a hemispherical electron analyzer (Vacuum Generators ADES 400) positioned at $45^{\circ}$ with respect to the sample normal. The analyzer was driven in the retarding mode with a constant resolution of $0.6 \mathrm{eV}$.

For hours, MDS spectra showed no change. This has been taken as proof of surface stability under beam exposure. An independent check of surface cleanliness was accomplished by UPS. Angle-resolved ultraviolet photoemission (AR-UPS) spectra taken before and after every MDS run showed no difference.

UPS measurements were taken with a He windowless differentially pumped discharge lamp emitting He I (21.2 $\mathrm{eV}$ ) and $\mathrm{He}$ II $(40.8 \mathrm{eV})$ photons. The incident angle on the sample, with respect to the surface normal, was set at $78^{\circ}$. Beam intensity was maintained at a constant value during measurements within $5 \%$ by monitoring the drain current on the sample holder.

\section{EXPERIMENTAL RESULTS AND DISCUSSION}

The experimental spectrum obtained for the GaAs(110) surface is shown in Fig. 2. Data showed repeatability on different cleavages.

Two broad structures can be identified, the first centered at about $7.7 \mathrm{eV}$ of kinetic energy and the second at about $2.3 \mathrm{eV}$. A well defined shoulder is present at about $3.5 \mathrm{eV}$ of kinetic energy. The high kinetic energy onset occurs at $11.6 \pm 0.2 \mathrm{eV}$, as determined by linear extrapolation on the experimental curve.

Both this value of the onset and shape clearly indicate the occurrence of a RI+AN deexcitation process. Moreover, it is important to stress the similarity with INS Auger neutralization results obtained on the same surface by Pretzer and Hagstrum (1966), using ions of $4 \mathrm{eV}$ of kinetic energy. ${ }^{11}$

On the basis of Eqs. (2) and (3), with $E_{i}=24.6 \mathrm{eV}$ (Fig. 1 ), with a photoelectric threshold value, $\phi=E_{\mathrm{gs}}+\chi$, of $5.5 \mathrm{eV}$ (Ref. 15) and with $E_{k}=11.6 \mathrm{eV}$, corresponding to electrons coming from the top of the valence band, a value of $\sim 1.5 \AA$ for $z_{0}$ can be estimated, in good agreement with values reported by other authors on metal and semiconductor surfaces. ${ }^{7,8,13}$

Because $\mathrm{RI}+\mathrm{AN}$ is the deexcitation mechanism, comparison of the experimental spectrum with theoretical predictions of the surface density of states can be done by performing a deconvolution operation on the experimental curve. To accomplish deconvolution, the experimental data were spline filtered (solid line in Fig. 2). The deconvolution method adopted is briefly described in the Appendix.

The result of deconvolution is presented in Fig. 3(a). The deconvoluted function is reported versus binding energy referred to the top of valence band, corresponding to the high kinetic energy onset of the electron distribution. Tests against mathematical artifacts are reported in the Appendix.

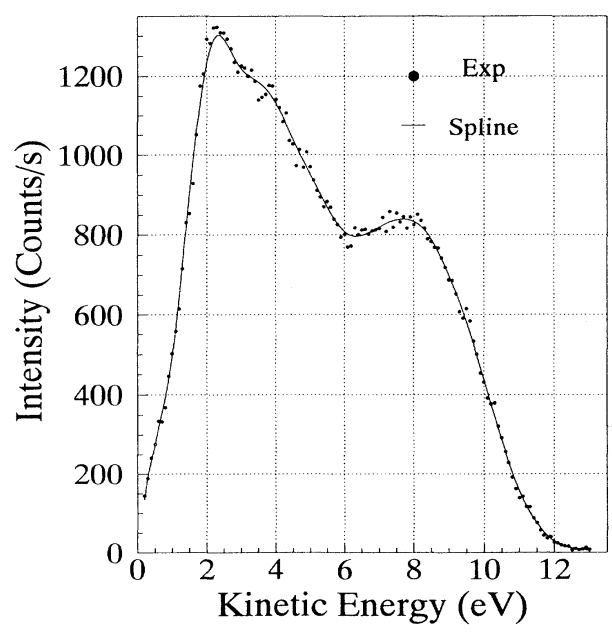

FIG. 2. Experimental (dots) and spline filtered (solid line) MD spectrum taken on the $\operatorname{GaAs}(110)$ surface. 


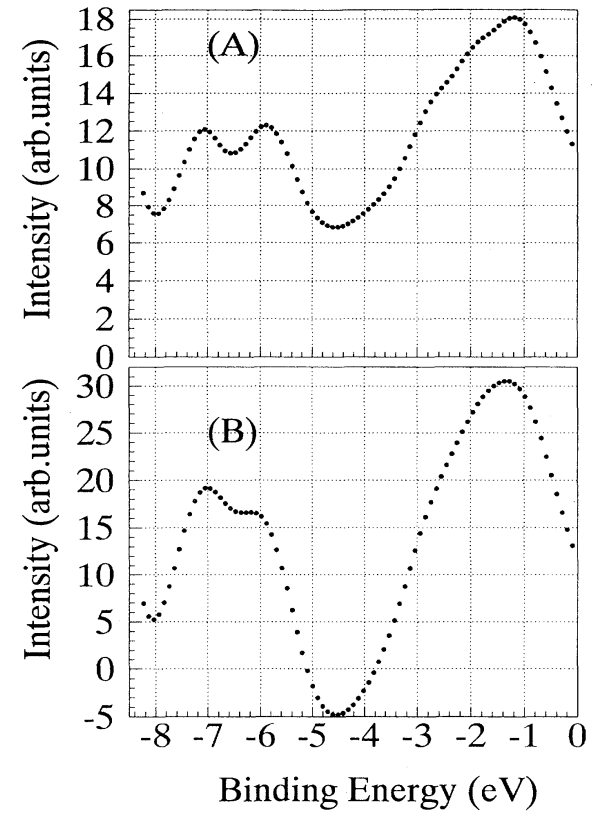

FIG. 3. (a) Deconvolution of the spline filtered MD spectrum versus binding energy. (b) First derivative of the spline filtered spectrum.

In Fig. 3(b), the first derivative of the filtered spectrum is presented. The derivative is known to provide, in a straightforward way, a first approximation to the deconvolution, though not reproducing the relative intensities. Moreover, it gives a good reliability test against the introduction of mathematical artifacts in the deconvolution procedure, as reported in the Appendix.

In both derivative and deconvolution, two broad structures are present, the first, higher in intensity, occurring between 0 and $4 \mathrm{eV}$ of binding energy and the second split in a doublet with structures at $5.9 \pm 0.2$ and $7.1 \pm 0.2 \mathrm{eV}$, respectively.

The first structure is centered at $1.2 \pm 0.2 \mathrm{eV}$ and presents a shoulder towards the higher binding energies. The question arises regarding the degree of confidence on the two peaks composing the doublet of the second structure. The doublet originates from faint slope variations in the spline filtered spectrum, which can be shown by taking the first derivative [see Fig. 3(b)]. However, it must be stressed that the doublet is independent of the filtering procedure adopted [different methods as fast Fourier transform (FFT) filtering, spline filtering, smoothing filtering were used giving essentially similar results] and from spectra taken on different cleavages.

The results of Figs. 3(a) and 3(b) have been obtained without any subtraction of the background of secondaries, which is substantially not known. The subtraction of a hypothetical structureless monotone background results in a reduction of the intensity of the deconvoluted spectrum with an increasing weight towards the higher binding energies. The subtraction is not shown in the present paper.

As a guide for better understanding the origin of the MDS observed structures, in addition to surface band structure calculations available in the literature, ${ }^{20-22}$ AR-UPS measurements were taken, in the same run, close to symmetry points of surface Brillouin zone (SBZ). AR-UPS results are summarized in Fig. 4; they have been published and discussed in Ref. 17. Features that can be ascribed to surface states have been detected and indicated on the experimental spectra, labeled $A_{5}, A_{4}$, $A_{3}, A_{2}^{\prime}, A_{1}^{\prime}$, and $C_{2}$ in the order. $A_{x}$ are states, whose charge density is predominantly localized on the anions (As atoms), $C_{x}$ are mainly localized on the cations ( $\mathrm{Ga}$ atoms). Primed labels refer to surface resonances.

The dispersion with $k_{\|}$shown by the experimental spectra is not an issue here and has already been discussed in Ref. 17. $A_{5}$ corresponds to a dangling bond state, mainly $p$ like in character, which is localized on the arsenic atom of the first atomic layer and protrudes mainly towards vacuum. ${ }^{21} A_{4}$ is a $p$-type back bond state, whose charge density is mainly localized between the first and the second layer. $A_{3}$ is a $p$-type state of the second layer. $C_{2}$, which is almost undetectable in UPS, is a $s$-type state localized on the gallium atom of the first atomic plane.

Moreover, a tight-binding calculation of the the density of states on different atomic planes has been accomplished. ${ }^{23}$ Tight-binding results are shown in Fig. 5 (only the densities of states relative to first and second layer are presented here). These results have been obtained choosing the surface relaxed geometry (Fig. 6) that gives a minimum in total energy calcula-

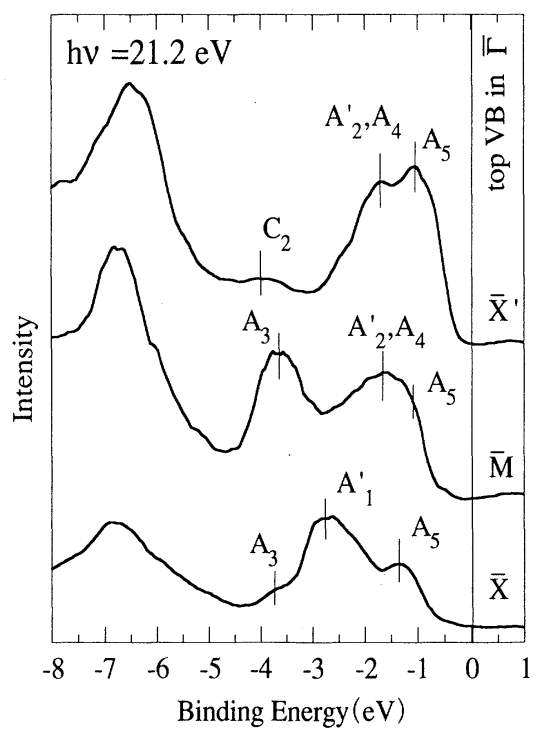

FIG. 4. UPS spectra (using $21.2 \mathrm{eV} \mathrm{He} \mathrm{I} \mathrm{photons)} \mathrm{taken} \mathrm{at}$ high symmetry points of SBZ (Ref. 17). The highest surface sensitivity is obtained at point $\bar{X}^{\prime}$ (grazing angle of electron collection). 


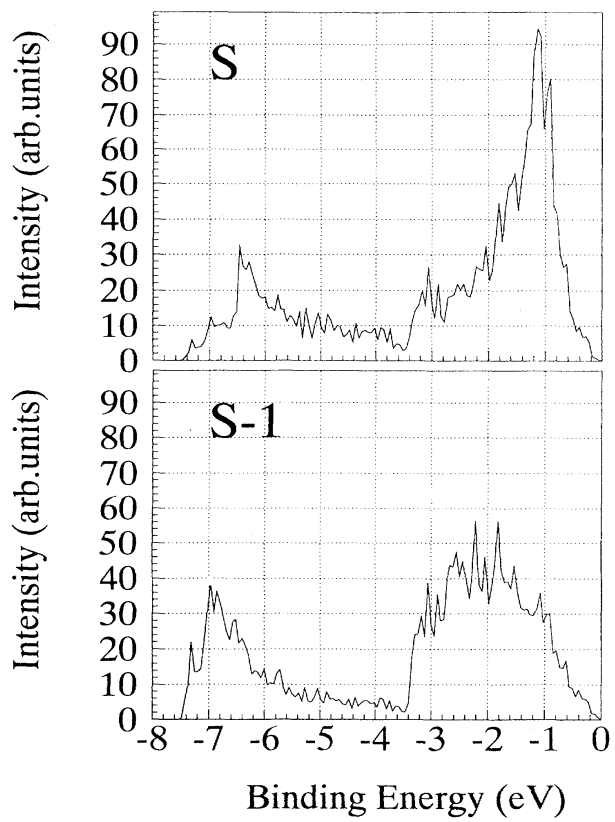

FIG. 5. First $(S)$ and second $(S-1)$ layer DOS obtained with tight-binding calculations, using slabs of 11 atomic planes (Refs. 23 and 25).

tions and agreement with low energy electron diffraction experiments. ${ }^{22}$ As will be seen in the following, the relaxed geometry gives also the best accordance with MDS results. The surface geometry parameters and the tightbinding parameters employed are those used in Ref. 22.

The assignment of the MDS observed structures is made on the basis of the comparison with theoretical results and photoemission spectra. At the same time, critically comparing UPS and MDS also leads to the possibility of drawing conclusions regarding the relative surface sensitivity of the two spectroscopies.

Starting from the first structure observed in deconvo-

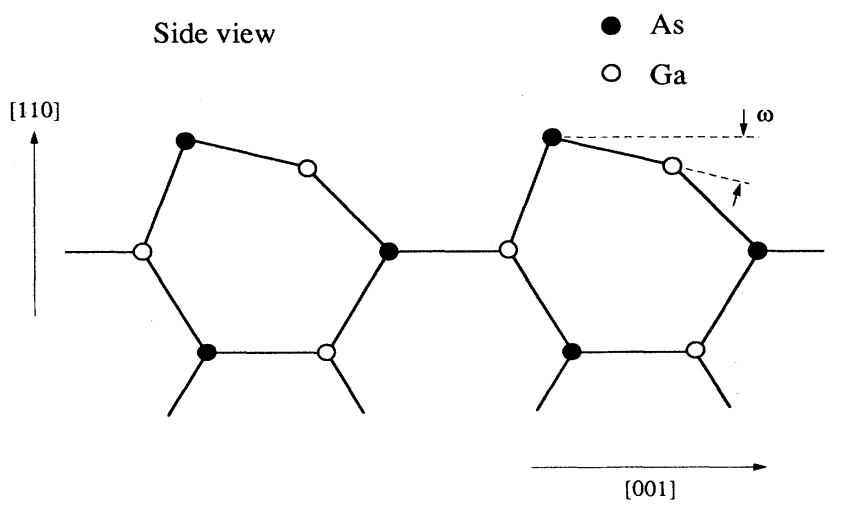

FIG. 6. Side view of the surface relaxed geometry of $\operatorname{GaAs}(110)$. Geometry parameters have been chosen according to Ref. 22, assuming a buckling angle $\omega=31.1^{\circ}$. lution with maximum at $1.2 \pm 0.2 \mathrm{eV}$, its energy is in good agreement with that of state $A_{5}$, obtained by theoretical calculations $^{20}$ (Fig. 5) and observed in AR-UPS measurements (particularly evident at point $\bar{X}^{\prime}$ of SBZ) (Fig. 4). $A_{5}$ gives the leading contribution in the MDS spectrum for at least two main reasons: $A_{5}$ shows the maximum of SDOS (top of Fig. 5); and, among the surface states, the SCD associated with it is the most protruding into vacuum. ${ }^{21}$

In the region between 0 and $4 \mathrm{eV}$ of binding energy, the asymmetry observed in the deconvolution structure towards the higher binding energies is also reproduced by the calculation. $A_{4}$ and $A_{3}$ states, besides $A_{1}^{\prime}$ and $A_{2}^{\prime}$ resonances, significantly contribute to this asymmetry. This is also evident in AR-UPS measurements, though with a completely different intensity ratio. Different from the first layer, the second layer DOS shows a pronounced feature close to the band edge and does not present peaked structures, as can be noticed from Fig. 5(S-1). The second layer DOS shows high density in the region between 2 and $4 \mathrm{eV}$ of binding energy, which could, in principle, contribute to the observed peak asymmetry. It could be interesting to evaluate the ratio between first and second layer contributions on the basis of a direct first principles simulation of the MDS process. This kind of analysis is out of the aim of present work, though theoretical work in this direction would be helpful.

The intensity minimum of the deconvolution function is centered at about $4.6 \mathrm{eV}$ of binding energy, in agreement with the position of the "stomach" gap of calculated surface band structure. ${ }^{20}$ Calculated local DOS shown in Fig. 5 present low density in the same energy position. Similar minima are present also in AR-UPS data, as shown in Fig. 4.

The second structure observed in deconvolution in the region between 5 and $8 \mathrm{eV}$ of binding energy is split into a doublet at $5.9 \pm 0.2$ and $7.1 \pm 0.2 \mathrm{eV}$. From the binding energy point of view, these two features can be assigned to surface states $C_{1}$ and $C_{2}$, which are responsible for structures in SDOS at these energies, as shown in Figs. $5(S-1)$ and $5(S)$, respectively. The assignment of the $7.1 \mathrm{eV}$ feature to $C_{1}$ deserves some caution, $C_{1}$ being assigned by theory to a cation $s$-like state principally localized on the second layer, ${ }^{21}$ with a surprisingly high MDS intensity relative to $C_{2}$ (assigned to the first layer). Background subtraction reduces the $C_{1}$ to $C_{2}$ branching ratio, though any background subtraction causes $C_{1}$ to disappear. The presence of a feature associated with $C_{1}$ in the experimental spectrum seems to indicate that this orbital too is at least partly involved in the deexcitation process. A first principle simulation of the MD spectrum on the $\operatorname{GaAs}(110)$ surface would be helpful in addressing this question.

AR-UPS results shown in Fig. 4 are of little help in such an assignment, since both $C_{2}$ and $C_{1}$ are mainly hidden by emission from bulk states. A weak feature, which can be associated with $C_{2}$, is visible only in spectra taken at grazing angles (close to point $\bar{X}^{\prime}$ of SBZ).

The above discussion on the weight of the states $C_{1}$ and $C_{2}$ in the MD spectrum and the discussion about 
the intensity ratio between $A_{5}$ and the group of states less surface localized $\left(A_{4}, A_{3}, A_{1}^{\prime}, A_{2}^{\prime}\right)$ give a measure of the relative surface sensitivity of MDS versus He I photoemission. Comparison leads to the conclusion that MDS is capable of giving a measure of the extent at which SCD protrudes into vacuum, while in UPS the degree of surface specificity is lower and in some cases severely obscured by bulk emission.

A further check on the assignment of the structures appearing in deconvolution can be made by observing the effects of hydrogen adsorption on $\operatorname{GaAs}(110) .{ }^{24}$ In particular, in agreement with experimental and theoretical results, ${ }^{17,25}$ hydrogen adsorption leads to a drastic reduction of the structure associated with $A_{5}$ in MDS. This gives us greater confidence in the results discussed in the present paper.

In conclusion, it is desirable to stress the similarities and differences between the physical information obtained by Bartynski et al. ${ }^{26}$ on $\operatorname{GaAs}(110)$, by Auger photoelectron coincidence spectroscopy (APECS), and by those obtained in the present study. Coincidence spectroscopy was employed to separate and study independently $\mathrm{Ga}$ and As $M_{4,5} V V$ Auger transitions. This allows us, in analogy with the present work, to obtain an effective SDOS. While in APECS case, this is obtained from separate projections on $\mathrm{Ga}$ and As sites, in the present case it results from simultaneous projection on the $\mathrm{He}$ atom.

\section{CONCLUSIONS}

A MDS study of $\operatorname{GaAs}(110)$ was presented. MDS was flanked by AR-UPS and experimental data were compared with theoretical results. To this end, a layer-bylayer tight-binding calculation was performed. Deexcitation was seen to proceed via RI+AN. An effective SDOS was obtained from the experimental spectrum through a deconvolution operation. The features of the deconvoluted spectrum were related to the first layer electronic structure and SCD.

The deconvoluted spectrum is dominated by two clearly distinct groups of structures. The low binding energy structure was ascribed mainly to emission from $A_{5}$, with lower contributions from other anion states, namely, $A_{4}, A_{3}, A_{1}^{\prime}, A_{2}^{\prime}$. $C_{1}$ and $C_{2}$ are responsible for the second group at higher binding energy. Theoretical work in the direction of a first principles simulation of the Metastable deexcitation process on the basis of a realistic surface band calculation would greatly enhance the power of the technique.

\section{ACKNOWLEDGMENTS}

The authors are pleased to acknowledge A. Plesanovas, A.C. Tarabini, and A. Ruocco for technical assistance and for their invaluable contribution to the experiment. F. Manghi is acknowledged for helpful discussion and for her contributions in layer-by-layer DOS calculation.

\section{APPENDIX}

\section{Deconvolution procedure}

Deconvolution was executed, following the method indicated by Boiziau et $a ._{.}{ }^{27}$ and Sesselmann et $a .^{13}$ The algorithm has been previously applied by Canepa et al. in Ref. 28. Schematically, the idea consists of building a "theoretical" SDOS function, $f(y)$, depending on a suitable number of parameters and in calculating a "theoretical" convolution, $S(y)=f(y) * f(y)$, to be compared with the experimental spectrum. The effective SDOS is obtained through a minimization procedure that permits determination of the unknown parameters. Minimization was accomplished using the MINUIT routine. ${ }^{29}$

Good results in the minimization procedure are obtained only if functions, which are smooth and free from rapid oscillations, are used. This implies a preliminary filtering of the experimental data. Low frequency noise was filtered out by taking the average of a suitable number of spectra ( 30 in present case), taken on the same cleavage surface. As mentioned in text, several filters were used to cut the high frequency noise: smoothing, FFT based filters, spline filtering. Best results have been obtained by using $b$-spline filtering. ${ }^{30}$ In Fig. 2, the av-

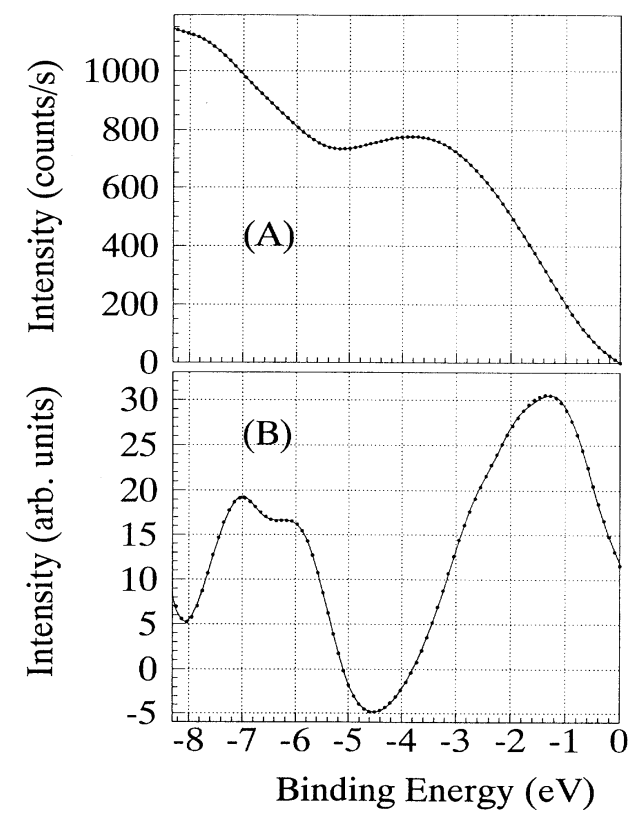

FIG. 7. (a) The backconvolution (dots) of the deconvolution shown in Fig. 2 is compared with the spline filtered spectrum (solid line). The good correspondence is index as the high reliability of the obtained result. A further check is shown in (b). Here, the first derivative of the dotted curve of (a) is compared with the first derivative of the spline filtered spectrum. Again a remarkable agreement can be found. 
erage experimental spectrum (dots) together with the spline filtered spectrum (solid line) are shown.

\section{Reliability tests}

Since mathematical artifacts can be introduced by data handling, it is necessary to be provided with suitable reliability tests. Concerning the deconvolution procedure, experience, together with previous works in literature ${ }^{13,31}$ shows that reliable results are reached only when the following conditions are satisfied. First, an optimum convergence of the minimization procedure must be achieved, giving a backconvolution of the deconvolution function, which is strictly adherent to the experimental data (the comparison is shown in Fig. 7, presenting an excellent correspondence). Moreover, $f(y)$ must fulfill two conditions, namely: (a) Function $f(y)$ must never be negative. (b) Structures present in deconvolution should also be present, though with different intensities, in the first derivative of filtered experimental spectrum..$^{31}$

The first derivative of filtered experimental spectrum is shown in Fig. 3(b). Although deconvolution and first derivative are based on completely different mathematical procedures, they show the same number of structures in exactly the same energy positions.
* Electronic address: pasquali@imoax1.unimo.it.

${ }^{1}$ G. Chiarotti, G. Del Signore, and S. Nannarone, Phys. Rev. Lett. 21, 1170 (1968); G. Chiarotti and S. Nannarone, Il Nuovo Cimento 39, 739 (1977); S. Nannarone, P. Chiaradia, F. Ciccacci, R. Memeo, P. Sassaroli, S. Selci, and G. Chiarotti, Solid State Commun. 33, 593 (1980).

${ }^{2}$ F. Houzay, G. Guichar, R. Pinchaux, G. Jezequel, F. Solal, A. Barsky, P. Steiner, and Y. Petroff, Surf. Sci. 132, 40 (1983); G.V. Hansson and I.G. Uhrberg, Surf. Sci. Rep. 9, 197 (1988); D.E. Eastman and W.D. Grobman, Phys. Rev. Lett. 28, 1378 (1972); L.F. Wagner and W.E. Spicer, Phys. Rev. B 9, 1512 (1974)

${ }^{3}$ S. D'Addato, S. Nannarone, and M. De Crescenzi, Surf. Sci. 162, 173 (1985), and references therein.

${ }^{4}$ W. Dose, H.J. Gossmann, and D. Straub, Surf. Sci. 117, 387 (1982); F.J. Himpsel, Appl. Phys. A 38, 205 (1985).

${ }^{5}$ S. Iacobucci, L. Marassi, R. Camilloni, S. Nannarone, and G. Stefani, Phys. Rev. B 51, 10252 (1995).

${ }^{6}$ A. Weiss, R. Mayer, M. Jibaly, C. Lei, D. Mehl, and K.G. Lynn, Phys. Rev. Lett. 61, 2245 (1988).

${ }^{7}$ H.D. Hagstrum, Phys. Rev. 96, 336 (1954).

${ }^{8}$ H.D. Hagstrum, Phys. Rev. 122, 83 (1961).

${ }^{9}$ W. Sesselmann, H. Conrad, G. Ertl, J. Küppers, B. Woratschek, and H. Haberland, Phys. Rev. Lett. 50, 446 (1983); Y. Harada, H. Ozaki, and K. Ohno, ibid. 52, 2269 (1984); J. Lee, C. Hanrahan, J. Arias, F. Boszo, R. Martin, and H. Metiu, ibid. 54, 1440 (1985); S. Nishigaki, N. Oishi, S. Matsuda, N. Kawanishi, and T. Sasaki, Phys. Rev. B 39, 8048 (1989); S. Masuda, H. Hayashi, and Y. Harada, J. Electron Spectrosc. Relat. Phenom. 51, 167 (1990); W. Maus-Friedrichs, M. Wehrhahn, S. Dieckhoff, and V. Kempter, Surf. Sci. 237, 257 (1990); M. Canepa, P. Cantini, L. Mattera, S. Terreni, and F. Valdenazzi, Phys. Scr. T41, 226 (1992).

${ }^{10}$ H.D. Hagstrum, Y. Takeishi, and D.D. Pretzer, Phys. Rev. 139, A526 (1965).

${ }^{11}$ D.D. Pretzer and H.D. Hagstrum, Surf. Sci. 4, 265 (1966).

${ }^{12}$ S. Masuda, H. Ishii, and Y. Harada, Surf. Sci. 242, 400 (1991).

${ }^{13}$ W. Sesselmann, B. Woratschek, J. Küppers, G. Ertl, and H. Haberland, Phys. Rev. B 35, 1547 (1987); 35, 8348 (1987).
${ }^{14}$ H.D. Hagstrum, in Chemistry and Physics of Solid Surfaces VII, edited by R. Vanselow and R. Rowe (Springer-Verlag, Berlin, 1988), p. 341.

${ }^{15}$ S.M. Sze, in Physics of Semiconductor Devices, 2nd ed. (Wiley, New York, 1982).

${ }^{16}$ E. Hood, F. Boszo, and H. Metiu, Surf. Sci. 161, 491 (1985).

17 A. Plesanovas, A. Castellani Tarabini, I. Abbati, S. Kaciulis, G. Paolicelli, L. Pasquali, A. Ruocco, and S. Nannarone, Surf. Sci. 307-309, 890 (1992).

${ }^{18}$ G. Paolicelli, G. Panaccione, R. Cosso, S. Kaciulis, S. Nannarone, I. Abbati, M. Canepa, S. Terreni, and L. Mattera, Advances in Surface Physics and Interfaces, Proceedings of the 17th Conference, Modena, 1992 [Il Vuoto 1, 106 (1994)].

${ }^{19}$ M. Canepa, C. Guarnascelli, L. Mattera, M. Polese, S. Terreni, and D. Truffelli, Rev. Sci. Instrum. 62, 1431 (1991).

${ }^{20}$ J.L.A. Alves, J. Hebenstreit, and M. Sheffler, Phys. Rev. B 44, 6188 (1991).

21 J.R. Chelikowsky and M.L. Cohen, Phys. Rev. B 20,4150 (1979).

22 C.A. Mailhiot, C.B. Duke, and C.J. Chadi, Surf. Sci. 149, 336 (1985).

${ }^{23}$ L. Pasquali and F. Manghi (unpublished).

${ }^{24}$ L. Pasquali, A. Plesanovas, A. Ruocco, A.C. Tarabini, S. Nannarone, I. Abbati, M. Canepa, L. Mattera, and S. Terreni, J. Electron Spectrosc. Relat. Phenom. 72,59 (1995).

${ }^{25}$ F. Manghi, C.M. Bertoni, C. Calandra, and E. Molinari, J. Vac. Sci. Technol. 21, 731 (1982).

${ }^{26}$ R.A. Bartynski, E. Jensen, K. Garrison, S.L. Hulbert, and M. Weinert, J. Vac. Sci. Technol. A 9, 1907 (1991).

${ }^{27}$ C. Boiziau, C. Garot, R. Nuvolone, and J. Roussel, Surf. Sci. 91, 313 (1980).

${ }^{28}$ M. Canepa, P. Cantini, L. Mattera, E. Narducci, M. Salvietti, and S. Terreni, Surf. Sci. 322, 271 (1995).

${ }^{29}$ F. James and M. Roos, Computer Code MINUIT, CERN, Geneva, Switzerland, 1988.

${ }^{30}$ R. Brun, O. Couet, C. Vandani, and P. Zanarini, Computer code PAW, CERN, Geneva, Switzerland, 1990.

${ }^{31}$ H.D. Hagstrum and G.E. Becker, Phys. Rev. B 4, 4187 (1971). 